\title{
ELECTROMAGNETIC FORCES IN INDUCTION MOTOR WITH NONSINUSOIDAL POWER SUPPLY
}

\author{
M.Roytgarts \\ Electrosila Stock Company \\ 139, Moskovsky pr., 196105, St-Petersburg, Russia \\ phone:+7 (812) 387 9754, fax:7 (812) 388 1814, e-mail: roitgarts@elsila.spb.ru
}

\begin{abstract}
Magnetic flux density and electromagnetic forces of induction motors at sinusoidal and nonsinusoidal power supply are analyzed. On the basis of asymptotic small parameter expansions depending on the level of higher time harmonics in the supply current waveform the most essential components of radial and tangential forces are singled out. With the given current waveform preliminary estimations of the relation of electromagnetic forces and torques of geometrically similar motors are obtained.

It is shown that even insignificant distortions in the supply current waveform may bear negative effect on increase in vibrations due to some additional torques and forces. These forces are conditioned by interaction of the main field and linear load waves from the current first and higher time harmonics. In relative units they are comparable with forces of interaction of the field tooth harmonics. Increase in the current harmonics leads to proportional increase in electromagnetic forces. At significant current harmonics some additional rise of the total level and enrichment of the spectrum of forces due to interaction of the field higher time harmonics between themselves takes place.
\end{abstract}

The computation results are compared with the experimental data for an induction motor power supplied from a pulse-width frequency converter.

\section{Key words}

Induction motor, nonsinusoidal current, electromagnetic forces.

\section{Introduction}

Nonsinusoidality of output currents of frequency converters saturates the induction motor magnetic field spectrum with additional space and time harmonics, increases additional torques, losses, vibrations and noises. The taking into account of the current higher harmonics makes the computation of electromagnetic forces and vibrations more complicated. There are a considerable number of publications dedicated to computations of electromagnetic forces both at sinusoidal and nonsinusoidal power supply [1]-[4]. However, the problem of force and vibration spectrum definition remains to be important. The task of comparative analysis and consideration of most essential components of forces is brought to the forefront.

This paper is aimed at a well-ordered analysis and comparison of magnetomotive forces (MMF), flux density and electromagnetic forces at sinusoidal and nonsinusoidal power supply of induction motor in steady state mode of operation.

Here we consider a problem in linear definition with the conventional assumptions for classical analysis of induction machines and restrict ourselves by the lower frequency area when we can neglect the damping of current higher harmonics of the stator due to eddy currents in the stator core.

In the paper asymptotic analysis techniques are used [5] and therefore some special terms shall be defined:

\section{List of special terms}

Quantity order - concept of asymptotic ratio between two functions, gives a quantitative characteristic of the upper boundary of this ratio.

Small parameter - dimensionless quantity which values are accepted to be of the same order with a relative quantity of amplitudes of higher space harmonics, the amplitude of the main harmonic is taken as a basic quantity.

Insignificant higher harmonics - quantities of the secondorder smallness relative to the main harmonic.

Small higher harmonics - quantities of the first-order of smallness relative to the main harmonic.

Significant higher harmonics - quantities comparative with the main harmonic. 


\section{Magnetic Field in Air Gap at Sinusoidal Power Supply}

With assumptions about infinite magnetic permeability of the core steel, small size of the air gap in comparison with a radius of the bore and pole division, rotating waves of the magnetic field radial induction in the asynchronous machine air gap can be presented as a product of harmonic expansions of MMF waves $F$ and air gap conductivity $\Lambda$

$$
B=F \cdot \Lambda \text {. }
$$

It is known that in ideally manufactured machines supplied with power from a symmetrical sinusoidal voltage source there are higher space harmonics of the magnetic field conditioned by discrete arrangement of windings and by air gap non-uniformity due to opening of the rotor and stator slots. Due to the designing measures taken, the amplitudes of these harmonics can be considered as quantities of the first-order smallness in comparison with the magnetic field main wave. The higher harmonic components of the rotor field, induced by higher space harmonics of the stator field, are ranked to the second-order smallness quantities because of significant leakage.

The asymptotic expansion of the resulting rotor and stator MMF can be presented as follows

$$
F=F_{0}+\varepsilon_{F}\left(\sum_{\nu \neq 1} F_{v}^{\prime}+\sum_{\mu \neq 1} F_{\mu}^{\prime}\right)+\varepsilon_{F}^{2} \sum_{\nu \neq 1} \sum_{\mu \neq 1} F_{\mu \nu}^{\prime}+o\left[\varepsilon_{F}{ }^{2}\right]
$$

where $F_{0}$ - the main harmonic of the resulting rotor and stator MMF,

$F_{v}$, - higher harmonics of the stator MMF,

$F_{\mu}, F_{\mu \nu}$ - higher harmonics of the rotor MMF induced by the main and higher harmonics of the stator field, respectively,

$\mathcal{E}_{F}$ - a small parameter specifying the order between the expansion members. The small parameter indicates a degree of quantity smallness not changing these quantities numerically, since

$$
\varepsilon_{F} \sum_{v} F_{v}^{\prime}=\sum_{v} F_{v}, \varepsilon_{F} \sum_{\mu} F_{\mu}^{\prime}=\sum_{\mu} F_{\mu}, \varepsilon_{F}^{2} \sum_{v} \sum_{\mu} F_{\mu \nu}^{\prime}=\sum_{v} \sum_{\mu} F_{\mu v}
$$

The asymptotic expansion can also be written for air gap magnetic conductivity at double-side teeth of the stator and rotor.

$$
\Lambda=\Lambda_{0}+\varepsilon_{\Lambda}\left(\sum_{i} \Lambda_{i}^{\prime}+\sum_{j} \Lambda_{j}^{\prime}\right)+\varepsilon_{\Lambda}^{2} \sum_{i} \sum_{j} \Lambda_{i}^{\prime} \Lambda_{j}^{\prime}+o\left[\varepsilon_{\Lambda}^{2}\right]
$$

where $\Lambda_{0}, \Lambda_{i}, \Lambda_{j}$ - magnetic conductivities of the uniform air gap, stator and rotor at single-side teeth.

Let's accept a ratio of order $\varepsilon_{F} \sim \mathcal{E}_{\Lambda} \sim \mathcal{E}$. To simplify the writing, we will leave out summarization signs in the future. Then, the asymptotic expansion of induction in air gap is obtained as

$$
\begin{aligned}
& B=B_{0}+\varepsilon\left(B_{v}^{\prime}+B_{\mu}^{\prime}+B_{z 1}^{\prime}+B_{z 2}^{\prime}\right)+\varepsilon^{2}\left(B_{v z 1}^{\prime}+\right. \\
& \left.+B_{v z 2}^{\prime}+B_{\mu z 1}^{\prime}+B_{\mu z 2}^{\prime}+B_{z 12}^{\prime}+B_{\mu \nu}^{\prime}\right)+o\left[\varepsilon^{2}\right]
\end{aligned}
$$

where $B_{0}=F_{0} \Lambda_{0}$ - main wave of induction in air gap, components in the first round brackets - first-order smallness winding and tooth harmonics of the stator and rotor field;

components in the second round brackets - second-order smallness interferential field harmonics arising from interaction of winding and tooth harmonics due to MMF and conductivity.

Expression (4) is the basis for computation of radial electromagnetic forces causing bending vibrations of the stator core at symmetrical sinusoidal power supply of the induction motor.

\section{Magnetic Field in Air Gap at Nonsinusoidal Power Supply}

The real current waveform when an electric motor is power supplied from a frequency converter has a polyharmonical character. Let's present the electric motor current phase as a Fourier series expansion

$$
i(t)=\sum_{k=1} i_{k} \cos \left(k \omega t-\varphi_{k}\right)
$$

where $i_{k}, \varphi_{k}, k \omega$ - amplitude, phase and circular frequency of higher time harmonics, $\omega$ - circular frequency of the main harmonic.

When current higher harmonic components flow through the stator winding, a complicated space-time spectrum of MMF and magnetic field in air gap, containing directly and reversely rotating waves is created. The quantity and direction of rotation of harmonics in three-phase machines are specified by the field generation factor

$$
\alpha_{k \gamma}=\frac{\cos \frac{2 \pi}{3}(k-v) \cdot \sin \pi(k-v)}{3 \cdot \sin \frac{\pi}{3}(k-v)}
$$

where $k, v$ - rotating wave time and space order, respectively.

In most simple case $k$ - integer, current contains higher time harmonics multiple to the main frequency, and it is followed from (6):

- $\quad$ if not one number in the pair $(k, v)$ is divisible by three, and $(k-v)$ is divisible by three, then directly and reversal rotating waves depending on sign $v$ are generated; 
- $\quad$ if only one number in the pair $(k, v)$ is divisible by three, then neither direct no reverse wave is available;

- $\quad$ if both numbers in the pair $(k, v)$ are divisible by three a pulsing field is created.

Table 1 shows as an example the direction of rotation of the field waves depending on the correlation of time and space harmonics orders for the machine with the stator symmetrical three-phase winding.

TABLE 1

\begin{tabular}{|c|c|c|}
\hline $\begin{array}{c}\text { ORDER OF } \\
\text { TIME } \\
\text { HARMONICS, } \mathrm{k}\end{array}$ & $\begin{array}{c}\text { ORDER OF } \\
\text { SPACE } \\
\text { HARMONICS, } v\end{array}$ & $\begin{array}{c}\text { DIRECTION OF } \\
\text { FIELD WAVES } \\
\text { ROTATIONS }\end{array}$ \\
\hline \multirow{2}{*}{1} & $\begin{array}{c}1,7,13,19,25, \ldots \\
-5,-11,-17,-23, \ldots\end{array}$ & $\begin{array}{c}\text { direct } \\
\text { reverse } \\
\text { pulsing }\end{array}$ \\
\hline \multirow{2}{*}{2} & $5,11,17,23,29, \ldots$ & direct \\
& $-1,-7,-13,-19,-25, \ldots$ & reverse \\
& - & pulsing \\
\hline \multirow{3}{*}{3} & - & direct \\
& $3,9,15,21,27, \ldots$ & reverse \\
& $1,7,13,19,25, \ldots$ & pulsing \\
\hline \multirow{2}{*}{4} & $-5,-11,-17,-23, \ldots$ & direct \\
& - & reverse \\
& pulsing \\
\hline & $5,1,17,23,29, \ldots$ & direct \\
& $-1,-7,-13,-19,-25, \ldots$ & reverse \\
& - & pulsing \\
\hline
\end{tabular}

Within the current spectrum of up-to-date frequency converters with pulse-width modulation method, contained are harmonics with frequencies proportional to both main frequency and carrier frequency and to their superposition. In this case it is necessary to go to the unified period of expansion or to the frequency to which both the main and carrier frequencies are multiple. The reference frequency is the highest common divisor of these frequencies. In such harmonic expansion the main harmonic is not already the first one. If we keep the main harmonic as the first one, expansion (5) will contain subdivided harmonics and subharmonics. In this case expression (6) for the field generation factor remains valid. Such an approach, accepted in the analysis of the field higher space harmonics [6], is used in this paper.

In practice induction motors are frequently power supplied from a voltage source. In this case the quantities of current higher harmonics are computed with the help of an equivalent circuit for induction motors, which takes into consideration higher space and time harmonics (Fig.1).

Here the slip is determined by the formula

$$
s_{k v}=1-(1-s) \frac{v}{k}
$$

where $s$ is rotor slip relative to the field main wave.

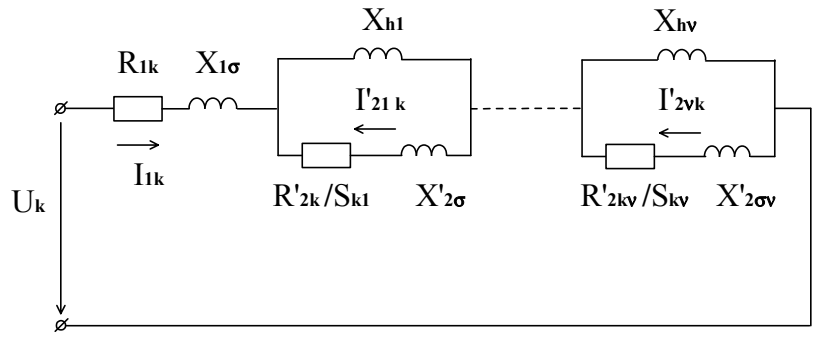

Fig. 1. Induction motor equivalent circuit at nonsinusoidal power supply

For comparative analysis of the stator current load within the time spectrum it is reasonable to single out small $(\sim \mathcal{E})$ and insignificant $\left(\sim \varepsilon^{2}\right)$ quantities of the harmonic components. The results are presented by the corresponding asymptotic expansion:

$$
A_{1(t)}=A_{1}+\varepsilon A_{k_{1}}^{\prime}+\varepsilon^{2} A_{k_{2}}^{\prime}+o\left[\varepsilon^{2}\right]
$$

where for the small parameter of expansion of time harmonics ratio of order $\varepsilon_{t} \sim \mathcal{E}$ is accepted, $A_{1}$ - stator current load at sinusoidal power supply, $A_{k_{1}}, A_{k_{2}}$ - the first and second order smallness higher time harmonics of the stator current load.

Let's present in the similar way the rotor and stator resulting MMF. For this we analyze preliminarily the equivalent circuit (Fig. 1). With the high orders of time harmonics and low orders of space harmonics, the slip quantity (7) differs slightly from unity, the operation mode is close to short-circuit, mutual-induction reactance is shunted by leakage reactance. In accordance with the equivalent circuit parameters, the ratio of magnetizing current to the stator current is considerably less for higher time harmonics than for the first one. In fact

$$
I_{0}=0\left[I_{1}\right], I_{0 k} \approx \frac{X_{2 \sigma}^{\prime}}{X_{h 1}+X_{2 \sigma}^{\prime}} I_{1 k}=o\left[I_{1 k}\right] \text {, }
$$

from which:

$$
\frac{I_{0 k}}{I_{0}}=\left(\frac{I_{0 k}}{I_{1 k}}\right)\left(\frac{I_{1 k}}{I_{1}}\right)\left(\frac{I_{1}}{I_{0}}\right)=\left(\frac{I_{1 k}}{I_{1}}\right) o\left[I_{1 k}\right] .
$$

It means that for higher time harmonics the order of smallness of magnetizing current relative to the stator current shall be increased. As a result for the current firstorder smallness higher harmonics, the main space wave of the resulting MMF of the rotor and stator has the second order smallness and it is comparable with MMF higher space harmonics at sinusoidal power supply. We obtain the asymptotic expansion of MMF as

$$
F_{t}=F+\varepsilon^{2} F_{k_{1}}^{\prime}+o\left[\varepsilon^{2}\right]
$$

where $F$ is asymptotic expansion of MMF (2) at monoharmonic sinusoidal power supply, 
$F_{k_{1}}^{\prime}$ are MMF higher time harmonics created by current load components $A_{k_{1}}^{\prime}$.

Let's multiply (10) by asymptotic expansion of conductivity of air gap (3) and single out additional to (4) components of the unified space-time expansion of the magnetic field induction in air gap.

$$
B_{n(t)}=B+\varepsilon^{2}\left(B_{0 k_{1}}^{\prime}+B_{v k_{1}}^{\prime}+B_{\mu k_{1}}^{\prime}\right)+o\left[\varepsilon^{2}\right]
$$

where $B$ is determined by expansion (4),

$$
B_{0 k l}=F_{0 k l} \Lambda_{0}, \quad B_{v k l}=F_{v k l} \Lambda_{0}, \quad B_{\mu k l}=F_{\mu k l} \Lambda_{0} .
$$

In the expansion for the magnetic field and for MMF, higher time harmonics of the main wave of the resulting field and higher space harmonics have the same order of smallness. For the main space field waves expression (11) becomes simplify:

$$
B_{n(t)}=B_{0}+\varepsilon^{2} B_{0 k_{1}}^{\prime}+o\left[\varepsilon^{2}\right] .
$$

Thus, if the current spectrum contains only insignificant higher time harmonics, then their effect on the radial component of the magnetic field in air gap can be neglected with accuracy up to the second-order smallness.

\section{Electromagnetic force density}

In accordance with the accepted assumption about infinite magnetic permeability of cores, electromagnetic forces act upon the stator bore surface on the air gap side. Having considered the density of magnetic forces as Maxwell Stress tensor, for the radial and tangential component we obtain:

$$
\begin{gathered}
\vec{T}_{n n}=\frac{1}{2 \mu_{0}}\left(B_{n}^{2}-B_{\tau}^{2}\right) \vec{n}, \\
\vec{T}_{n \tau}=\frac{1}{\mu_{0}} B_{n} B_{\tau} \overleftarrow{\tau}
\end{gathered}
$$

where $B_{n}, B_{\tau}$ - radial and tangential components of the magnetic field induction, $n, \tau$ - orts in the radial and tangential direction.

In formula (12) the square of the induction tangential component is negligibly small in comparison with the square of radial component in asynchronous machines with a small-sized air gap $\delta$, when the ratio $2 p v \delta / D<1$

is fulfilled. Here $p-$ number of the pole pairs, $D-$ diameter of the stator core.

Moreover, with infinite permeability of the core, the magnetic field intensity tangential component on the bore surface $H_{\tau}$ is equal to the stator linear current load $A_{l}$. For computation of the force density applied to the stator surface the well-known expressions are valid

$$
\begin{gathered}
\vec{T}_{n}=\frac{1}{2 \mu_{0}} B_{n}^{2} \vec{n}, \\
\vec{T}_{\tau}=B_{n} A_{1} \vec{\tau} .
\end{gathered}
$$

Let's determine the density of electromagnetic forces with the help of obtained above asymptotic expansions of electromagnetic fields at sinusoidal and nonsinusoidal power supply. In this case we restrict ourselves by forces with a small number of waves (low space orders) with the amplitudes being no less than the second-order smallness. In doing so, we take into consideration that dynamic bends of the stator caused by tangential forces are considerably lower than bends due to radial forces. In this connection for the tangential forces we restrict ourselves to zero-form forces caused by interaction of the main space waves of linear load and induction.

\section{A. Sinusoidal power supply}

When determining the density of radial electromagnetic forces from expression (12a), let's make use of asymptotic expansion of induction at sinusoidal power supply (4). As a result we obtain the improved expansion:

$$
\begin{aligned}
T_{n} & =\frac{1}{2 \mu_{0}}\left\{B_{0}^{2}+\varepsilon 2 B_{0} B_{v}^{\prime}+\varepsilon^{2}\left[2 B_{0} B_{\mu v}^{\prime}+2 B_{v}^{\prime} B_{\mu}^{\prime}+\right.\right. \\
& \left.\left.+4 B_{v}^{\prime} B_{z 2}^{\prime}+4 B_{\mu}^{\prime} B_{z 1}^{\prime}+4 B_{z 1}^{\prime} B_{z 2}^{\prime}\right]+o\left[\varepsilon^{2}\right]\right\} .
\end{aligned}
$$

In order to obtain radial force waves of the low space forms we take into account higher harmonic inductions of orders $\mu$ and $v$, which algebraic sum module is minimal. In this connection, for example, the second composed of expression (14) is taken into account only for lower orders of the winding harmonics of bipolar machines. The forces conditioned by interaction of spatial harmonics of the tooth and winding order have the second-order of smallness relative to the force determined by the main induction wave square. In the accepted definition of the problem, zero-form and periodically time-dependent tangential forces in an electric motor with sinusoidal power supply are not available. However, these forces appear when unbalance of sinusoidal phase currents is taken into account.

\section{B. Nonsinusoidal power supply}

Let's consider additional electromagnetic forces at nonsinusoidal power supply depending on a quantity of higher harmonics in the supply current waveform.

\section{1) Insignificant current higher harmonics}

In a practically sinusoidal network the stator current higher harmonics are usually insignificant and make up quantities of the second-order smallness relative to the main frequency harmonic. It was above established that radial components of the magnetic field produced from these current harmonics turned out to be quantities less than those of the second-order smallness and they are not 
included in expansion (11). In this connection the components of the forces determined from (11a) turned out to be also negligibly small.

Additional components of the zero-form tangential forces determined from (8) and (11a) are of the following view,

$$
T_{\tau}^{\prime}=\varepsilon^{2} B_{0} A_{k 2}^{\prime}+o\left[\varepsilon^{2}\right]
$$

where the main space waves of induction and higher time harmonics of the stator linear load are determined by expressions

$$
\begin{gathered}
B_{0}=B_{0 m} \cos \left(\omega t-p \varphi-\phi_{0}\right), \\
A_{k_{2}}=A_{k_{2} m} \cos \left(k_{2} \omega t \mp p \varphi-\phi_{k_{2}}+\pi / 2\right) .
\end{gathered}
$$

It follows from (15) that the additional space zero-form tangential forces have frequencies $f_{R}=f\left(k_{2} \mp 1\right)$. Here the upper sign corresponds to the directly rotating waves, the lower sign - to inversely rotating waves.

It is seen from comparison of (14) and (15) that in relative units tangential forces arising from supply current higher harmonics insignificant by value have the same order of smallness as radial forces arising from the field higher space harmonics at induction motor sinusoidal power supply. However, when determining vibrations caused by these forces one should take into account the difference in the stator dynamic properties between bending and torsion vibrations.

\section{2) Current small higher time harmonics}

This is the most typical case when induction motors are power supplied from frequency converters (Fig. 2). Higher time harmonics of the converter output currents in relative units are comparable with the field higher space harmonics at sinusoidal power supply.

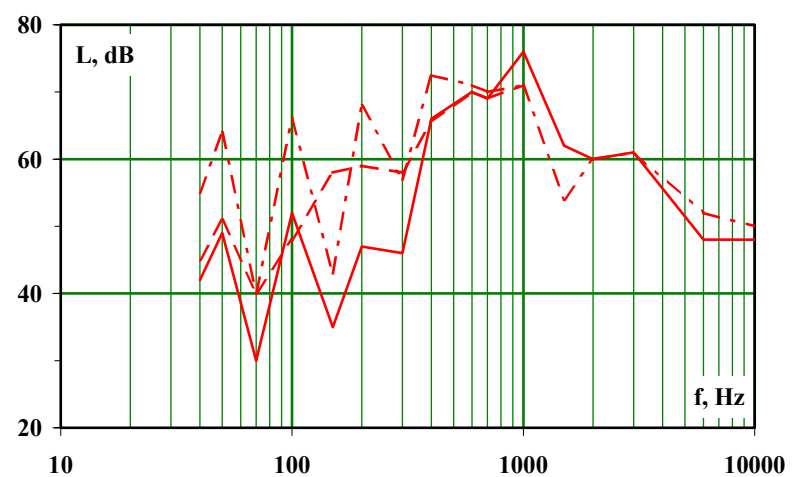

Fig. 2. Electric motor vibrations versus thyristor commutator operation mode

The density of additional radial electromagnetic forces arising from these current harmonics is equal

$$
T_{n}^{\prime \prime}=\left\{\varepsilon^{2}\left(B_{0} B_{0 k_{1}}^{\prime}+B_{0}^{\prime} B_{v k_{1}}^{\prime}\right)+o\left[\varepsilon^{2}\right]\right\} / \mu_{0}
$$

where higher time harmonics of the field main space wave and of the stator field winding harmonics are determined by the following expressions

$$
\begin{aligned}
B_{0 k_{1}} & =B_{0 k_{1} m} \cos \left(k_{1} \omega t \mp p \varphi-\phi_{0 k_{1}}\right), \\
B_{v k_{1}} & =B_{v k_{1} m} \cos \left(k_{1} \omega t \mp \nu p \varphi-\phi_{k_{1}}\right) .
\end{aligned}
$$

The both components (16) have the amplitudes being comparable in relative units with the amplitudes of forces arising as a result of interaction of the field higher space harmonics at sinusoidal power supply (14). The first component $T_{n}^{\prime \prime}$ has space forms $R=p \mp p$ and frequencies $f\left(k_{l} \mp 1\right)$, the second component $T_{n}^{\prime \prime}$ has space forms $R=p|v \mp 1|$ and frequencies $f\left(k_{l} \mp 1\right)$. The identical signs in the expressions for forms and frequencies are accepted at interaction of waves with the same direction of rotation, the opposite signs - at interaction of waves with the opposite direction of rotation. To obtain low forms, it is reasonable to consider the second component (16) only for the field lower space harmonics of bipolar machines.

Additional components of the density of tangential forces are determined by expression

$$
T_{\tau}^{\prime \prime}=\varepsilon B_{0} A_{k_{1}}^{\prime}+\varepsilon^{2} B_{0 k_{1}}^{\prime} A_{1}+o\left[\varepsilon^{2}\right],
$$

where

$$
A_{1}=A_{1 m} \cos \left(\omega t-p \varphi-\phi_{1}+\pi / 2\right) .
$$

These forces have a zero form and frequencies $f_{R}=f\left(k_{1} \mp 1\right)$. The density of tangential forces arising from interaction of the field main wave with the stator linear load higher time harmonics in relative units exceeds by an order the density of radial forces arising from interaction of the magnetic field higher space harmonics at sinusoidal power supply. Moreover, tangential forces of the second-order smallness arising from interaction of the field main wave higher time harmonics with the first harmonic of the stator linear load are added.

\section{3) Significant current higher harmonics}

Unless accepted special engineering solutions are accepted the amplitudes of the current fifth time harmonic may account $20 \%$, of the seventh $-14 \%$ of the first one. The value of the converters with pulse-width modulation current higher harmonics may be even greater. These harmonics are significant. Increased content of higher harmonics in the supply current waveform leads to proportional rise of electromagnetic forces causing vibrations. Furthermore, the force frequency spectrum gets rich due to interaction of higher time harmonics of the induction and of linear load between themselves. 


\section{Assessment of Forces and Moments of Geometrically Similar Electric Motors}

At the design stage a preliminary assessment of electromagnetic forces and torques causing vibrations should be done on the basis of the prototype data [7]-[9]. If in the process of designing the conditions of geometrical similarity are fulfilled and, in addition, the supply frequency converter output current waveform is kept unchangeable, then transferring from tangential forces to alternating-sign torques, we obtain a dependence on the stator current harmonic composition in absolute units:

$$
M_{\tau}=\frac{M_{n}}{\cos \varphi}\left(\alpha_{k} \frac{I_{1 k}}{I_{1}}\right)
$$

where $M_{n}$ and $\cos \varphi$ are induction motor rated torque and power factor.

It follows that torques acting upon the stator at nonsinusoidal power supply are proportional to the rated torque, and for machines with equal speed of rotation - to the machine capacity. At the same voltage for two identical machines the following is true

$$
\frac{M_{\tau(1)}}{M_{\tau(2)}}=\left(\frac{\alpha_{k(1)} I_{1 k(1)}}{\alpha_{k(2)} I_{1 k(2)}}\right)
$$

i.e. the torques are proportional to the relationship of supply current higher harmonics.

Let's also express radial forces through the main induction wave

$$
T_{n}=B_{0}^{2}\left(\alpha_{k} \frac{I_{0 k}}{I_{0}}\right) / \mu_{0}
$$

Within a series of machines, induction in air gap is practically unchangeable. In small-power machines the relative value of no-load current varies depending on power in proportion $P^{-1 / 7}$, a fraction of higher harmonics of the magnetizing current and a quantity of electromagnetic forces rise, respectively. For large-sized machines the no-load current relative value is practically independent of power. It follows that when geometrically similar large-sized induction motors are power supplied from frequency converters with stable current waveform, a density of additional radial forces is also independent of power. At a change of the relative quantity of higher harmonics in the current waveform, both the torques and radial forces vary proportionally.

Figure 3 illustrates the spectrum of vibrations of two geometrically similar electric motors within a series.

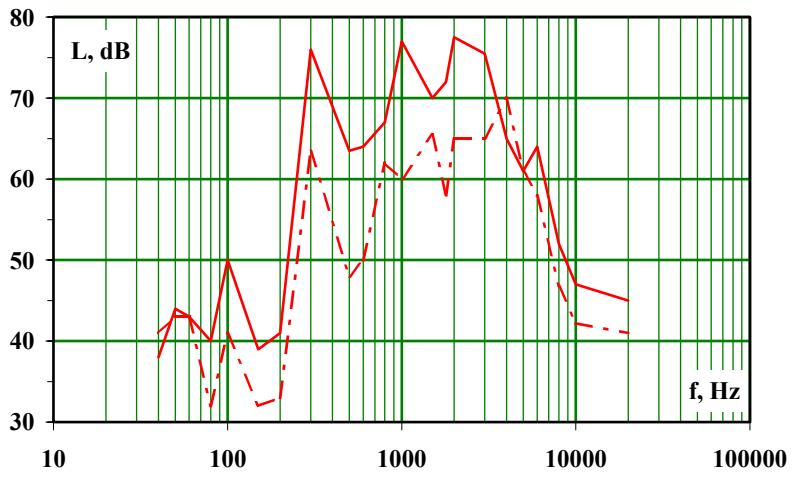

Fig. 3. Vibration levels of production induction motors A 71-4 $(-)$ and A 52-4 (-・-・-) when power supplied from a frequency converter with a d.c. link

\section{Efficiency of Measures on Reduction of Electromagnetic Forces}

Shortening of the winding pitch as well as a skewing of the stator and rotor slots reduces forces generated from interaction of higher space harmonics of the stator and rotor fields [10], however, it does not practically affect the forces due to interaction of the field "long" waves which are the main sources of vibrations at nonsinusoidal power supply.

Use of the rotor closed slots as well as use of massive rotors decrease the radial induction components and additional radial forces from higher time harmonics, but does not practically tell upon tangential forces and torsion torques.

In the same time it turns to be effective to increase the number of phases of the induction motor and frequency converter as well as improvement of the output current waveform. It is reasonable to shift the higher harmonic spectrum to the area of high frequencies as well as to shift as much as possible between the current higher harmonic frequencies.

It is expedient to increase the stator rigidity relative to the bending and zero-form torsion vibrations to reduce vibrations caused by electromagnetic forces.

\section{Computation Example}

Let's consider the spectrum of current supplying of an induction motor from a frequency converter with a carrier frequency of $330 \mathrm{~Hz}$ and rated frequency of $18.9 \mathrm{~Hz}$ (Fig. 4). In this case the reference frequency makes up $0.3 \mathrm{~Hz}$. According to the given analysis we consider the main harmonic to be the first one and use fractional value of $k$.

Table 2 shows the relative values of the ten current harmonics and relevant values of the field generation factor $\alpha_{\mathrm{k}}$. 


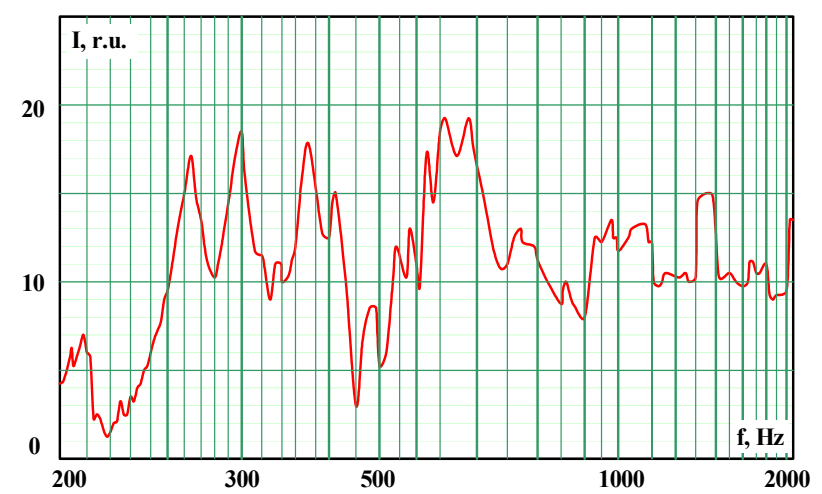

Fig.4. Supply current spectrogram

Table 2 - Current higher harmonics and field generation factors

\begin{tabular}{|c|c|c|c|}
\hline & & \multicolumn{2}{|c|}{$\alpha_{\mathrm{k}}$} \\
\cline { 3 - 4 } FREQUENCY, & CURRENT & DIRECT & REVERCE \\
$\mathrm{Hz}$ & $\mathrm{I}_{0 \mathrm{k}} / \mathrm{I}_{01}, \mathrm{p} . \mathrm{u}$. & FIELD & FIELD \\
\hline 264 & 0.355 & 0 & 1 \\
\hline 300 & 0.446 & 1 & 0 \\
\hline 335 & 0.078 & 0.251 & 0.003 \\
\hline 372 & 0.4 & 0.05 & 0.76 \\
\hline 411 & 0.2 & 0.9 & 0.17 \\
\hline 540 & 0.08 & 0.17 & 0.52 \\
\hline 580 & 0.141 & 0.67 & 0.28 \\
\hline 660 & 0.531 & 0 & 1 \\
\hline 690 & 0.531 & 0.33 & 0.33 \\
\hline 770 & 0.126 & 0.12 & 0.67 \\
\hline
\end{tabular}

The computation results of radial electromagnetic forces are given in Table 3 .

Table 3 - Force components conditioned by nonsinusoidal power supply

\begin{tabular}{|c|c|c|c|}
\hline $\begin{array}{c}\text { FORCE } \\
\text { COMPONENTS, } \\
\text { p.u. }\end{array}$ & \multicolumn{2}{|c|}{$\begin{array}{c}\text { CURRENT AND } \\
\text { FIELD } \\
\text { FREQUENCIES, Hz }\end{array}$} & $\begin{array}{c}\text { FORCE } \\
\text { FREQUENCIES } \\
\text { Hz }\end{array}$ \\
\hline 0.199 & 18.9 & 264 & 283 \\
\hline 0.25 & 18.9 & 300 & 281 \\
\hline 0.17 & 18.9 & 322 & 391 \\
\hline 0.3 & 18.9 & 660 & 679 \\
\hline 0.098 & 18.9 & 690 & 709 \\
\hline 0.098 & 18.9 & 690 & 671 \\
\hline 0.011 & 18.9 & 335 & 316 \\
\hline 0.1 & 18.9 & 411 & 392 \\
\hline 0.019 & 18.9 & 411 & 430 \\
\hline 0.008 & 18.9 & 540 & 521 \\
\hline 0.023 & 18.9 & 540 & 559 \\
\hline 0.053 & 18.9 & 580 & 561 \\
\hline 0.022 & 18.9 & 580 & 599 \\
\hline 0.009 & 18.9 & 770 & 751 \\
\hline 0.047 & 18.9 & 770 & 799 \\
\hline
\end{tabular}

Fig. 5 gives the spectrogram of point acceleration on the electric motor housing surface at power supply from a frequency converter with a pulse-width modulation in relative units. The computation results compared with the experimental data show that almost all the force components listed in Table 3 with the exception of the forces with small magnitude result in vibrations in electric motors.



Fig.5. Vibration spectrum of electric motor at nonsinusoidal current, shown in Fig. 4

\section{Conclusion}

It is shown that even insignificant distortions of the supply current waveform may be source of the additional vibrations in induction motors, since the space zero-order excess tangential forces torques and are appeared. They are produced from interaction of the first and higher time harmonics main space waves of field components. In relative units these forces are comparable with forces of interaction of the field tooth harmonics at sinusoidal power supply (the tooth forces). The current first-order smallness time harmonics produce some additional radial electromagnetic forces, which are comparable with the tooth forces, and additional tangential forces, considerably exceeding the tooth forces. Further increase in higher time harmonics within the current spectrum leads to proportional increase in electromagnetic forces. Moreover, the forces arising from interaction of higher time harmonics of the field radial and tangential components between themselves should be taken into account. The situation becomes aggravated if the electromagnetic force frequencies are close to resonance frequencies of the corresponding forms of the stator.

In relative units the density of additional tangential forces exceeds by an order the density of radial forces, however, the both components shall be considered because of different dynamic properties of the stator for bending and torsion vibrations. For geometrically similar machines with the power raise the alternating-sign torsion moments increase in proportion. In the same time the density of radial forces with the power rise remains practically unchangeable if the supply current waveform is kept unchangeable. This property may be used for diagnostics of effect of radial and tangential forces on vibrations of induction motor.

To reduce vibrations when motor is power supplied from frequency converters, it is reasonable to improve the current waveform either directly in the converters or with the help of external filters, to shift the higher harmonic spectrum to the area of high frequencies as well as to shift as much as possible the frequency spectrum components between themselves. Also, it is effectively to use multiphase induction motors, to increase the stator rigidity relative to bending and torsion vibrations. 


\section{References}

[1] Jordan H., Der gerauscharme elektromotor. W.Girardet, Essen, 1950, s.100.

[2] Yang S.J., Timar P.L., The effect of harmonic currents on the noise of a three-phase induction motor. IEEE Transactions on PAS. Vol. PAS-99, 1, pp.307-310, 1980.

[3] Shubov I.G., The noise and vibration of electric machines, Leningrad, 1986, pp.208.

[4] Belmans R., Hameyer K., Impact of inverter supply and numerical calculation techniques in audible noise problems, in Proc. International seminar on vibrations and acoustic noise of electric machinery, Bethune, 25-26 May 1998.

[5] Nayfeh A.H. Perturbation methods. John Wiley and Sons, 1973, pp.455.

[6] Richter R. Elektrische maschinen, Bd.4, Verlag Springer, Berlin, s.471, 1936.
[7] Schuisky W. Berechnung der elektrischen Maschinen. Berlin, 1960, pp.731.

[8] Roytgarts M.B. Electromagnetic compatibility and vibration activity of electric machines, In book: Theses of reports of 1 International conference on electromechanotronics EMT97, St. Petersburg, 1997.

[9] Petrov G.P., Roytgarts M.B. About possibility of similarity methods application for preliminary estimate of regulated electric drives vibration, Electrotechnica, №11, pp.27-33, 1998.

[10] Roytgarts M.B. Vibration exciting forces and moments at mutual skewing of stator and rotor slots, in Proc. International seminar on vibrations and acoustic noise of electric machinery, Bethune, France, 25-26 May 1998. 